\title{
A case report of high-grade astroblastoma in a young adult
}

\author{
Sagar Bhalerao*,1, Rajnish Nagarkar ${ }^{2}$ \& Aditya Adhav ${ }^{2}$ \\ ${ }^{1}$ Department of Medical Oncology, HCG Manavata Cancer Centre, Nashik, India \\ ${ }^{2}$ Department of Surgical Oncology, HCG Manavata Cancer Centre, Nashik, India \\ *Author for correspondence: academics@manavatacancercentre.com
}

\begin{abstract}
Astroblastoma is an uncommon neuroepithelial primary tumor of the brain which is of uncertain origin. We present a case of high-grade astroblastoma in an 18-year-old female with a severe headache, loss of appetite, vomiting and generalized weakness. The patient had undergone a right frontoparietal craniotomy. Large subfalcine meningioma was excised. The lesion was suspected to be a meningioma. Primary radiological investigation revealed a $6.8 \mathrm{~cm} \times 5.8 \mathrm{~cm} \times 5.4 \mathrm{~cm}$ lesion. Although the radiological and intraoperative findings were of an extra-axial tumor, the histology and immunophenotype was of an astroblastoma. The patient was treated with cyclophosphamide, cisplatin and etoposide chemotherapy regimen. The patient was later treated with bi-weekly bevacizumab. The patient had improved symptomatically post-chemotherapy. However, there was no significant difference in lesion size. The patient died after 2 weeks. The prognosis of patients with astroblastoma is extremely poor as observed in our case.
\end{abstract}

First draft submitted: 9 October 2018; Accepted for publication: 20 December 2018; Published online: 28 February 2019

Keywords: astroblastoma $\bullet$ brain neoplasm $\bullet$ brain tumor $\bullet$ magentic resonance imaging

Astroblastoma is an uncommon neuroepithelial primary tumor of the brain which is of uncertain origin. Astroblastoma is commonly observed in the cerebral hemisphere of children and young adults. As per current evidence, the incidence of astroblastoma ranges between 0.45 and 2.8 percent among all neuroglial tumors [1]. The diagnosis of astroblastoma is challenging as it is rarely encountered in clinical practice. The histopathologic and radiological appearances often resemble other glial neoplasms [1]. Astroblastomas are solid, peripheral and large brain tumors. Common clinical symptoms include a headache, vomiting, nausea, seizures and decay in consciousness levels [2]. Although astroblastomas are considered as pediatric tumors, there are several cases observed in young adults as well as older patients. The tumor is commonly observed in the frontoparietal hemispheres. Some of the other locations include the cerebellum, brainstem, intraventricular and hypothalamus. Astroblastomas are uncommon tumors of the central nervous system, wherein their classification, diagnosis, histogenesis and management are still disputed [3]. The World Health Organization (WHO) had previously not classified astroblastoma due to the absence of sufficient clinicopathological data. However, the most recent update labels astroblastoma as a high-grade (Grade 4) neuroepithelial tumor with unknown origin [3]. Radical surgical resection can be considered as the treatment of choice in such cases. In the case of high-grade lesions, radiotherapy may play a key adjuvant role. The role of chemotherapy for astroblastoma remains unclear [4]. We present a case of high-grade astroblastoma in an 18-year-old female.

\section{Case report}

An 18-year-old female patient reports to the clinic with a severe headache, loss of appetite, vomiting and generalized weakness for 15 days. The patient had undergone a right frontoparietal craniotomy. Large subfalcine meningioma was excised. The lesion was suspected to be a meningioma. A histopathological correlation was awaited.

A follow-up magnetic resonance imaging (MRI) was carried out after 5 months. Noncontrast MRI of the brain was performed with 5-mm-thick T1W, T2W, fluid-attenuated inversion recovery (FLAIR), 5-mm-thick

Future Medicine 
(A)

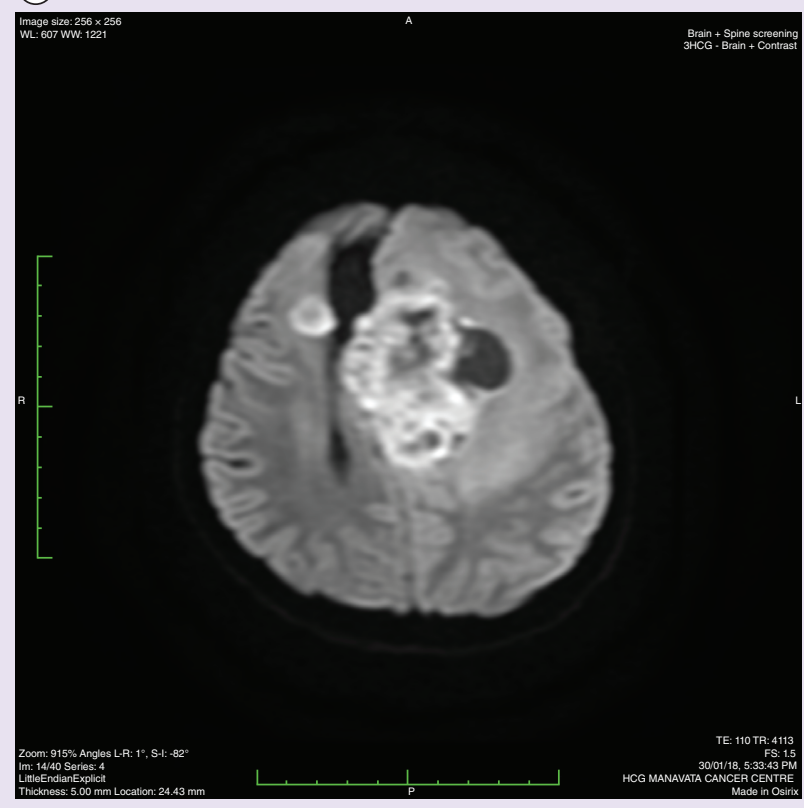

(c)

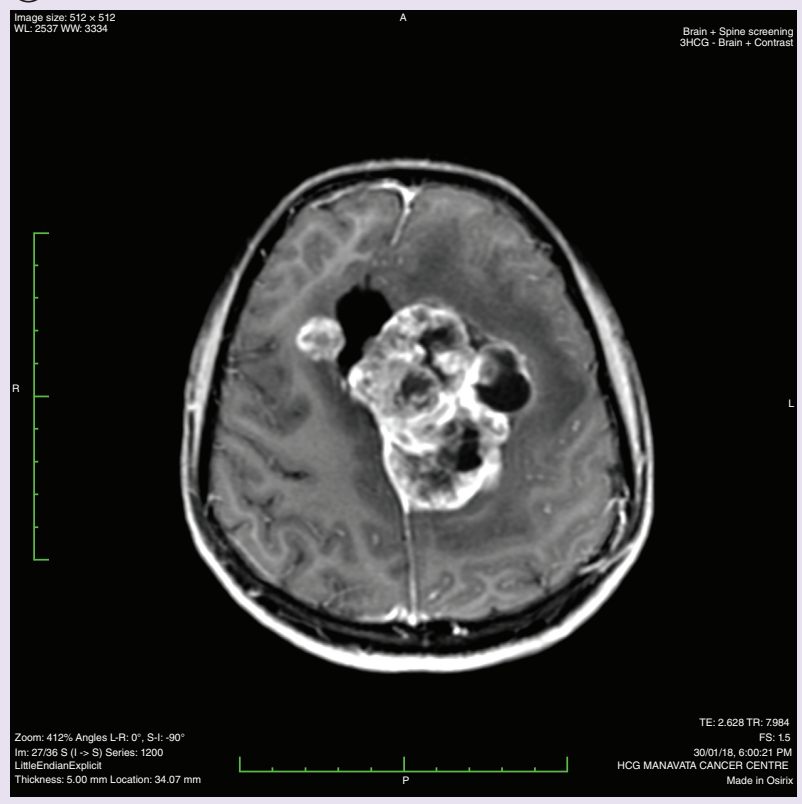

Figure 1. Radiological findings. (A) A large, well-defined, irregular and heterogeneously enhancing mass lesion involving the cortical and subcortical region was observed. The lesion was $6.8 \mathrm{~cm} \times 5.8 \mathrm{~cm} \times 5.4 \mathrm{~cm}$ in size. (B) A marked perilesional white matter edema in the left frontal region and the left frontotemporal region was observed. (C) It appears hyperintensive on T2W and FLAIR images.

T2W coronal images and 5-mm-thick T1W sagittal images. MRI contrast was performed. MR spectroscopy and perfusion was also performed.

As per the radiological findings, a large, well-defined, irregular and heterogeneously enhancing mass lesion involving the cortical and subcortical region was observed (Figure 1A). The lesion extended from the left frontoparietal region with a few petechial hemorrhages within the contrast-enhancing areas. The lesion appeared hypointense to gray matter on T1, hyperintense on T2W and FLAIR images (Figure 1B \& C). On T1C contrast images, the 
lesion appeared to have heterogeneous contrast enhancement. A cystic area measuring $19 \times 15 \mathrm{~mm}$ was observed adjacent to the lesion.

The lesion was $6.8 \mathrm{~cm} \times 5.8 \mathrm{~cm} \times 5.4 \mathrm{~cm}$ in size (Figure 1A). A marked perilesional white matter edema in the left frontal region and the left frontotemporal region was observed. It appears hyperintensive on T2W and FLAIR images and shows restricted water motion of diffusion-weighted (DW) images. It has marked signal intensity on ADC images which favors vasogenic edema. A hyperenhancing well-defined lesion measuring $15 \times 12 \mathrm{~mm}$ was also observed in the right centrum semiovale.

The gradient echo images show that there are a few petechial hemorrhages within the contrast-enhancing areas. Bordering these petechial hemorrhages are areas of solid tissue that demonstrate relatively restricted diffusivity compared with the normal white matter.

The moderate mass effect was observed over the left lateral ventricle and a midline shift of $23 \mathrm{~mm}$ was noted.

MR perfusion-derived relative cerebral blood volume (rCBV) maps demonstrate heterogeneous perfusion abnormalities. The solid contrast enhancing tissue shows relatively higher normalized rCBV ratios in comparison with white matter, while the nonenhancing T2 hyperintense tissue and other areas demonstrate relatively lower normalized rCBV ratios. The maximum normalized rCBV ratio calculated by placing multiple regions of interest measure up to 1.52 .

There was a marked increase in the choline:creatinine (Cho/Cr) and choline: $\mathrm{N}$-acetyl aspartate (Cho/NAA) ratios while a marked decrease in $\mathrm{Cho} / \mathrm{Cr}$ and $\mathrm{Cho} / \mathrm{NAA}$ ratio was observed. Abnormal metabolites were observed in the bed that include lipids and lactate. In addition, a small dural-based hyperenhnaicng lesion measuring $6 \mathrm{~mm}$ in the right inferior frontal region was observed. It resembled small meningioma. Rest of the ventricles and basal cisterns appear normal. Rest of the cerebral sulci are unremarkable. Visualized major intracranial arteries at the skull base show normal flow voids.

On immunohistochemistry, the tumor showed focal weak positivity for glial fibrillary acidic protein. Occasional tumor cells showed positivity for epithelial membrane antigen. However, the tumor was negative for neuronal nuclei (NeuN), Synaptophysin, Mic-2 and p53 protein. The MIB-1 labeling index was approximately $15-20 \%$ in the focal highest proliferating areas. The overall features were clearly indicative of high-grade astroblastoma.

Although the radiological and intraoperative findings were of an extra-axial tumor, the histology and immunophenotype was of an astroblastoma, which in all probability arose in an extremely superficial location with dural involvement.

Based on the opinion of our multidisciplinary team that included a neurosurgeon, the patient's lesion was considered inoperable. In order to avoid any permanent disability, the lesion was not surgically resected. The patient was closely monitored throughout the chemotherapy regimen. Surgical resection was kept as an option post-chemotherapy.

\section{Treatment}

The patient was treated with cyclophosphamide + cisplatin + etoposide regimen. The patient was later treated with bi-weekly bevacizumab. There is no standard chemotherapy protocol for patients presenting with astroblastoma.

\section{Follow-up MRI post-chemotherapy}

A follow-up MRI was performed to evaluate disease progression post-chemotherapy. The radiological findings were as follows: a large well-defined irregular shaped altered signal intensity lesion is noted in the left frontoparietal and left capsule-ganglionic region showed a mild increase in bulk as compared with the previous scan. The lesion was $7.5 \mathrm{~cm} \times 5.5 \mathrm{~cm} \times 5.19 \mathrm{~cm}$ in size (Figure 2A). Small mass lesion in the right centrum semiovale was observed as in the previous scan (Figure 2B \& C). A marked perilesional white matter edema in the left frontal region and the left frontotemporal region was observed. The moderate mass effect was observed over the left lateral ventricle and a midline shift of $21 \mathrm{~mm}$ was noted. There was a marked increase in the $\mathrm{Cho} / \mathrm{Cr}$ and Cho/NAA ratios.

The patient had improved symptomatically post-chemotherapy. However, there was no significant difference in the lesion size. The patient died after 2 weeks.

\section{Discussion}

Astroblastoma is an extremely uncommon glial tumor that is predominantly observed in the cerebral hemispheres of young adults. The histogenesis of astroblastoma to date remains to be debated. It is difficult to determine the number of astroblastoma cases reported in the literature. However, it is estimated that astroblastoma accounts for 
(A)

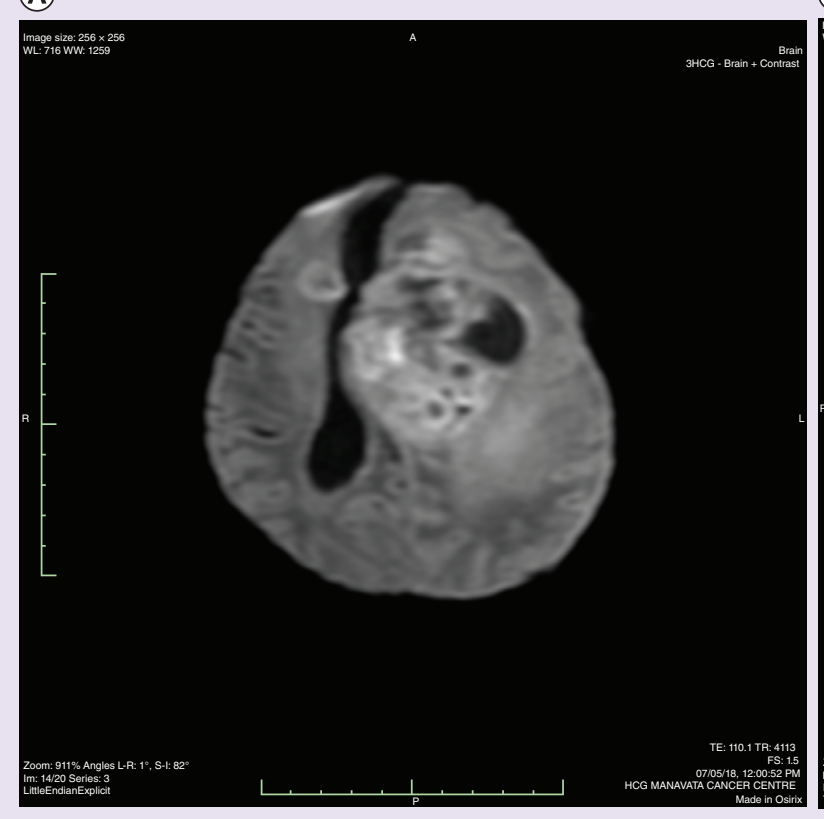

(B)



(c)

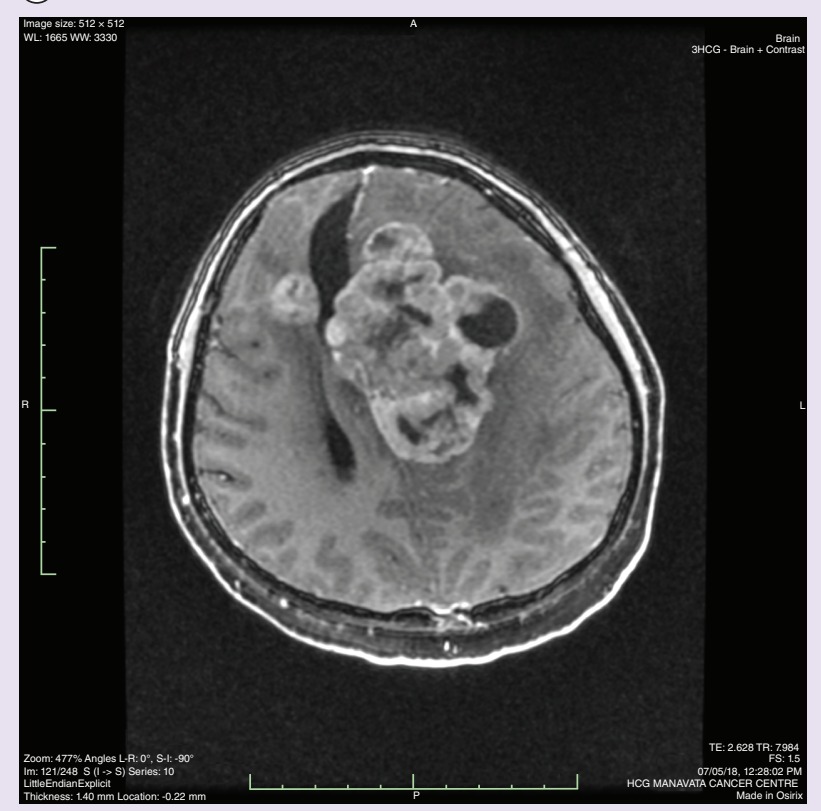

Figure 2. Radiological findings. (A) A large well-defined irregularly shaped altered signal intensity lesion is noted in the left frontoparietal and the left capsule-ganglionic region showed a mild increase in bulk as compared with the previous scan. The lesion was $7.5 \mathrm{~cm} \times 5.5 \mathrm{~cm} \times 5.19 \mathrm{~cm}$ in size. (B; C) Small mass lesion in the right centrum semiovale was observed as in the previous scan.

approximately $0.45-2.8 \%$ of all neurological tumors [5]. Astroblastoma remains an uncommon clinical entity with an unknown cellular origin. Variable clinical outcomes in patients with astroblastoma make it a highly debatable concept. The term 'astroblastoma' was used by Bailey and Cushing as a different type of glioma in 1924. They categorized the entity as an exclusive form of astrocytic glioma [6].

The term 'astroblastoma' is confusing since the tumor is neither astrocytic nor is it 'blastic'. The tumor cells of astroblastoma were found to be an intermediate between astrocytes and ependymal cells as per an electron 
microscopy. Astroblastoma is believed to have originated from the astroblast which is an intermediate between glioblasts and astrocytes [7].

Astroblastomas can occur at any age. However, based on bimodal age distribution, astroblastomas are common or at peak during infancy (5-10 years) and in young adults (21 and 30 years). Females are commonly diagnosed with astroblastoma with the male-to-female ratio reported at 1:11 [8].

As per our knowledge and current literature, astroblastoma is predominantly observed in the cerebral hemispheres. However, other locations could include the corpus callosum, pineal gland, cerebellum and the brain stem [9]. The cardinal symptoms of astroblastoma include headache and neurological defects as observed in our case [9].

The typical radiological appearance of astroblastoma represent FLAIR images, heterogeneous hyperintense signal on T2-weighted sequences (T2WS), and hypointense to isointense on T1-weighted sequences (T1WS), as observed in our case [10]. Typical appearances also include heterogeneous enhancement on contrast-based T1WS images as seen in our case [10].

There is no standard treatment for astroblastoma due to its rarity and difficulty in early-stage clinical diagnosis. However, surgery continues to remain the primary management approach for this condition. In the case of lowgrade lesions, complete resection may be curative. In the case of high-grade astroblastomas, patients have a poor prognosis due to the invasion of local brain regions, high recurrence rates and rapid progression. In such cases, aggressive treatment and continuous follow-up are mandated. In our case, a high-grade astroblastoma was confirmed on histopathology, while surgical resection was avoided as per the neurosurgeon's recommendations. The presence and extent of peritumoral T2 hyperintensity or peritumoral edema on MRI can be considered as a feature for predictive recurrence of the tumor [11].

Radiation therapy (RT) has evolved as a mainstay treatment for low-grade tumors. In a recent study, patients who did not receive RT had a poor survival compared with those who received RT. Although the results of the study were not statistically significant, RT was found to be clinically beneficial for patients with high-grade astroblastoma. Recurrence in astroblastoma is mainly local; however, distant CNS metastasis has also been observed. Appropriate radiation field for astroblastoma remains unclear and needs further investigation [12].

As per the NCCN Clinical Practice Guidelines in Oncology for central nervous system cancers, cisplatin + etoposide + cyclophosphamide can be used as an effective systemic treatment approach for patients with brain tumors [13]. As per a recent meta-analysis, the combination of chemotherapy along with bevacizumab was attributed with progression-free survival and object response rate. However, there was no significant impact or prolongation in overall survival [14]. Thus, in our case the patient was treated with bi-weekly bevacizumab.

The prognosis of astroblastoma can be predicted by the extent of resection and histology findings. The prognosis of low-grade astroblastoma is similar to that of low-grade gliomas. The prognosis of high-grade astroblastoma is similar to that of anaplastic astrocytomas considering the rate of progression and recurrence [15]. Unusual cases such as anaplastic astroblastoma and astroblastoma with bone invasion have also been reported in the literature $[16,17]$.

In one of the most interesting cases, long-term remission was observed in a 50-year old Caucasian female with astroblastoma. The patient was treated with palliative intent. The patient was treated with procarbazine/lomustine $(\mathrm{CCNU}) /$ vincristine (PCV) course. Chemotherapy was attributed with a complete radiological response. The patient was free of disease 7 years after surgery and 6 years after chemotherapy. Although there is no standard treatment regimen for such patients, chemotherapy proved to improve survival [18].

In one of the largest patient series for astroblastoma $(n=239)$, researchers assessed key prognostic factors. Treatment, supratentorial location and older age were poor prognostic factors for astroblastoma patients prior to 1990 [19]. It was also thought that patients with cerebellar tumors had a better prognosis by having early signs of increased intracranial pressure. This helped in faster diagnosis and effectively timely treatment as compared with those with supratentorial tumors. They also indicated that patients diagnosed after 1990 had better prognosis due to advances in diagnostic and therapeutic approaches such as MRI [19]. Molecular classification of central nervous system tumors have also been helpful in differentiating different tumor entities. For example, the CNS HGNET-MN1 was found to closely represent astroblastoma [20]. Further molecular sequencing analysis can help differentiate and better understand the molecular characteristics of astroblastoma.

\section{Conclusion}

Astroblastoma is an extremely rare primary brain tumor with several diagnostic and therapeutic challenges. Due to the low incidence of the disease, examining tumor characteristics and developing effective treatment regimens is 
a major challenge. Astroblastoma has an unpredictable biological course with an increased risk of recurrence and rapid progression.

\section{Future perspective}

Astroblastoma is an uncommon brain tumor with no standard line of treatment. Although patients with lowgrade astroblastoma can be surgically resected, chemotherapy is an ideal treatment approach in cases of high-grade astroblastoma. Case reports like these help disseminate knowledge on uncommon tumors like astroblastoma with insights on diagnostic and treatment challenges. Although case reports are considered at the lowest in the evidence hierarchy, they would help researchers conduct large clinical studies in the future. These studies may help understand key histopathological features and treatment options for astroblastoma.

\section{Summary points}

- Astroblastoma is characterized with unpredictable clinical behavior with limited information on its management.

- Gross total resection has been associated with increased survival. However, the role of adjuvant radiation or chemotherapy remains controversial.

- Treatment for astroblastoma should be considered with a curative intent since many patients become long-term survivors.

- Patients with high-grade tumors are most likely to receive radiation and/or chemotherapy.

- Large clinical studies need to be carried out with a comprehensive pathologic review. It would help determine the association between prognosis and histopathological features.

- Adjuvant chemotherapy in astroblastoma is not supported to date.

- Patients with high-grade tumors who do not receive adjuvant radiation are expected to have poor prognosis.

- The role of chemotherapy or radiation after surgical resection needs further investigation.

Financial \& competing interests disclosure

The authors have no relevant affiliations or financial involvement with any organization or entity with a financial interest in or financial conflict with the subject matter or materials discussed in the manuscript. This includes employment, consultancies, honoraria, stock ownership or options, expert testimony, grants or patents received or pending, or royalties.

Acknowledgment

Writing assistance was provided by Lyndon Fernandes.

Open access

This work is licensed under the Attribution-NonCommercial-NoDerivatives 4.0 Unported License. To view a copy of this license, visit http://creativecommons.org/licenses/by-nc-nd/4.0/

\section{References}

Papers of special note have been highlighted as: $\bullet$ of interest

1. Agarwal V, Mally R, Palande DA, Velho V. Cerebral astroblastoma: a case report and review of literature. Asian J. Neurosurg. 7(2), 98-100 (2012).

- The article is one of the very few articles based in India with a case of astroblastoma in a young patient. The case not only provides key radiological characteristics but also a histopathological correlation on astroblastoma.

2. Sadiq M, Ahmad I, Shuja J, Ahmad Z, Ahmed R, Ahmad K. Astroblastoma in a young female patient: a case report and literature review of clinicopathological, radiological and prognostic characteristics and current treatment strategies. Brain Tumor Res. Treat. 5(2), 120-126 (2017).

- A case similar to our case but with an exceptional insight on key radiopathological characteristics of astroblastoma. The authors provided a comprehensive review on astroblastoma with key focus on treatment strategies and prognostic factors.

3. Samples DC, Henry J, Bazan C, Tarasiewicz I. A case of astroblastoma: radiological and histopathological characteristics and a review of current treatment options. Surg. Neurol. Int. 7(Suppl. 40), S1008-S1012 (2016).

- An unusual case wherein a 30-year-old patient was diagnosed with astroblastoma. The patient underwent gross total resection and adjuvant radiotherapy as in our case. The report helped us rationalize the need for surgery and adjuvant radiotherapy.

4. Salvati M, D’Elia A, Brogna C et al. Cerebral astroblastoma: analysis of six cases and critical review of treatment options. J. Neurooncol. 93(3), 369-378 (2009). 
- One of the very few case series published in the literature. The authors have provided key insights in the classification, diagnosis, histogenesis and therapeutic management of astroblastoma. An interesting fact is the use of temozolomide (TMZ) chemotherapy. Of the six cases, three patients with low-grade astroblastoma were alive at follow-up (for 2, 15, and 19 years), which was remarkable.

5. Barakat MI, Ammar MG, Salama HM, Abuhashem S. Astroblastoma: case report and review of literature. Turk Neurosurg. 26(5), 790-794 (2016).

6. MacKenzie DJ. A classification of the tumours of the glioma group on a histogenetic basis with a correlated study of prognosis. Can. Med. Assoc. J. 16(7), 872 (1926).

7. Kim DS, Park SY, Lee SP. Astroblastoma: a case report. J. Korean Med. Sci. 19(5), 772-776 (2004).

8. Hammas N, Senhaji N, Alaoui Lamrani MY et al. Astroblastoma - a rare and challenging tumor: a case report and review of the literature. J. Med. Case Rep. 12(1), 102 (2018).

9. de la Garma VH, Arcipreste AA, Vázquez FP, Aguilar RR, Castruita UO, Guerra RM. High-grade astroblastoma in a child: report of one case and review of literature. Surg. Neurol. Int. 5, 111 (2014).

10. Singh DK, Singh N, Singh R, Husain N. Cerebral astroblastoma: a radiopathological diagnosis. J. Pediatr. Neurosci. 9(1), 45-47 (2014).

- The case report was based on different symptoms, in other words, the patient presented with generalized tonic-clonic seizures and intracranial tension. The patient was quite young while the authors provided key insights on the surgical management of this case. A brief review on astroblastoma and its current treatment approaches were also discussed.

11. Payne C, Batouli A, Stabingas K et al. A pediatric tumor found frequently in the adult population: a case of anaplastic astroblastoma in an elderly patient and review of the literature. Case Rep. Neurol. Med. 2017, 1607915 (2017).

12. Merfeld EC, Dahiya S, Perkins SM. Patterns of care and treatment outcomes of patients with astroblastoma: a National Cancer Database analysis. CNS Oncol. 7(2), CNS13 (2018).

- An interesting paper on the outcomes of patients with astroblastoma treated with specific chemotherapy regimens. It is an important paper in the field of neuro-oncology since it helps clinicians and oncologists understand patient outcomes post-radiotherapy. It helped us analyze the role of radiotherapy in astroblastoma.

13. NCCN Clinical Practice Guidelines in Oncology ${ }^{\top M}$. Central Nervous System Cancers. v 1.2012. www.alabmed.com/uploadfile/2014/0515/20140515070238502.pdf

14. Yang S-B, Gao K-D, Jiang T, Cheng S-J, Li W-B. Bevacizumab combined with chemotherapy for glioblastoma: a meta-analysis of randomized controlled trials. Oncotarget 8(34), 57337-57344 (2017).

- The article is of significant importance not only to the medical literature but also to our case. We treated our patient with bevacizumab. This is the only meta-analysis on bevacizumab for glioblastoma in the literature, as per our knowledge. As per the report, bevacizumab was found to improve progression-free survival and object response rate.

15. Hammas N, Senhaji N, Alaoui Lamrani MY et al. Astroblastoma - a rare and challenging tumor: a case report and review of the literature. J. Med. Case Rep. 12(1), 102 (2018).

16. Binesh F, Akhavan A, Navabii H, Mehrabaniyan M. Anaplastic astroblastoma: a rare glial tumour. BMJ Case Rep. 2011:bcr0620114323 (2011).

17. Bhattacharjee S, Pulligopu AK, Uppin MS, Sundaram C. Astroblastoma with bone invasion. Asian J. Neurosurg. 6(2), 113-115 (2011).

18. Bergkåsa M, Sundstrøm S, Gulati S, Torp SH. Astroblastoma - a case report of a rare neuroepithelial tumor with complete remission after chemotherapy. Clin. Neuropathol. 30(6), 301-306 (2011).

19. Ahmed KA, Allen PK, Mahajan A, Brown PD, Ghia AJ. Astroblastomas: a Surveillance, Epidemiology, and End Results (SEER)-based patterns of care analysis. World Neurosurg. 82(1-2), e291-e297 (2014).

20. Sturm D, Orr BA, Toprak UH et al. New brain tumor entities emerge from molecular classification of CNS-PNETs. Cell 164(5), 1060-1072 (2016).

- It is one of the very few research papers on molecular classification of rare brain tumors. Considering that the future of cancer treatment would be based on molecular genetics or precision medicine, this paper is of significant importance. It helped us understand how classification of brain tumors may help define new treatment modalities. 
